\title{
TRAF1-ALK fusion indicates poor prognosis to ALK tyrosine kinase inhibitors in relapsed/refractory ALK-positive anaplastic large-cell lymphoma patients
}

\section{Diwen Pang}

Guangdong General Hospital

\section{Ling Huang}

Guangdong General Hospital,Guangdong Academy of Medical Sciences https://orcid.org/0000-00025373-7394

\section{Xinmiao Jiang}

Guangdong General Hospital

\section{Feili Chen}

Guangdong General Hospital

Hanguo Guo

Guangdong General Hospital

\section{Xiaojuan Wei}

Guangdong General Hospital

Sichu Liu

Guangdong General Hospital

\section{Yan Teng}

Guangdong General Hospital

\section{Zhanli Liang}

Guangdong General Hospital

Wenyu Li ( $D$ lwy80411@163.com )

https://orcid.org/0000-0002-6444-7149

\section{Research article}

Keywords: Anaplastic large cell lymphoma\Anaplastic lymphoma kinase inhibitors, Next-generation sequencing

Posted Date: July 7th, 2020

DOI: https://doi.org/10.21203/rs.3.rs-39803/v1 
License: (c) (i) This work is licensed under a Creative Commons Attribution 4.0 International License. Read Full License 


\section{Abstract \\ Background}

Relapsed/refractory (R/R) anaplastic lymphoma kinase (ALK)-positive anaplastic large cell lymphoma (ALCL) respond to ALK inhibitors, but resistance bears a poor prognosis. No biomarkers predict a long duration of response to ALK inhibitors. The ALK gene was first identified as the fusion partner of the nucleophosmin (NPM1) gene in recurrent $t(2 ; 5)($ p23;q35) found in an ALCL subset. However, several distinct ALK fusions that result in highly different characteristics have also been described in lymphomas.

\section{Methods}

We retrospectively reviewed 43 patients with pathologically confirmed ALK-positive ALCL at Guangdong Provincial People's Hospital from February 2007 through February 2020, including seven R/R patients who received ALK inhibitors (six with crizotinib and one with alectinib). We performed next-generation sequencing (NGS) with paraffin-embedded tissue for these seven R/R patients and one patient with a peripheral blood sample. We evaluated clinical characteristics and survival status.

\section{Results}

The median age of all patients was 29 (range 15-66) years. Most patients were male with advanced stage and B symptoms. The 5-year progression-free survival (PFS) and overall survival (OS) rates were $60 \%$ and $70 \%$, respectively with a median follow-up of 52.2 (range 2.4-168.6) months. Multivariate analyses revealed that only bone marrow involvement was an independent prognostic factor for PFS ( $P=$ 0.03 ) and $O S(P=0.03)$. Of the seven R/R patients, the median line number of therapies was four (range $3-7)$ and that of ALK inhibitor usage was three (range 2-5). The overall response rate was $100 \%(n=7)$. The NGS identified four patients with the NPM1-ALK fusion and two with tumor necrosis factor receptorassociated factor 1 gene (TRAF1)-ALK fusion; the latter quickly developed resistance to chemotherapy and ALK inhibitors.

\section{Conclusions}

ALK inhibitors improved survival of patients with R/R ALK-positive ALCLs. TRAF1-ALK fusion may predict a poor clinical outcome to chemotherapy and ALK inhibitors. This ALK fusion may reflect a trend toward the aggressive behavior of lymphomas.

\section{Introduction}


Anaplastic large-cell lymphoma (ALCL), characterized by CD30 + pleomorphic large lymphocytes, compromises approximately $1-4.4 \%$ of non-Hodgkin lymphomas and encompasses a heterogeneous subtype of diseases ${ }^{12}$. The 2016 World Health Organization (WHO) classification recognizes three types of systemic anaplastic large cell lymphomas: ALK-positive ALCL (ALK + ALCL), ALK-negative ALCL (ALK$A L C L)$ and breast implant-associated $A L C L^{3}$. $A L K+A L C L$ is recognized as a distinct entity and is generally characterized by a unique chromosomal translocation involving the ALK gene on chromosome $2 p 23$ and the nucleophosmin (NPM) gene on chromosome 5q35, forming a novel chimeric fusion protein, NPM-ALK, which is represented in $75-85 \%$ of all ALK $+\mathrm{ALCL}^{4}$. Other partner chromosomes have also been identified, all resulting in overexpression of the ALK protein and occasionally different immunohistochemical staining patterns ${ }^{5}$. Up to $66 \%$ of ALCL patients are ALK + ALCL, which often occurs more frequently in young male patients with a median age of 30 years old, and is usually present at an advanced stage, including $B$ symptoms and extranodal site involvement ${ }^{26-8}$.

CHOP (cyclophosphamide, doxorubicin, vincristine, and prednisone) or CHOP-like regimens remain the standard of care for ALK + ALCL. ALK + ALCL has a more favorable prognosis in comparison with ALKALCL, particularly in younger patients with a low International Prognostic Index (IPI) score. The 5-year overall survival (OS) rate for ALK + ALCL ranges from $67-80 \%^{27-9}$, which does not rule out confounding factors that can potentially influence the prognosis, such as age and ALK gene fusion type. The prognostic significance of ALK expression remains controversial because of the unsatisfying response to standard therapy in some ALK + ALCL patients, which is likely to reflect genetic heterogeneity. About 45$65 \%$ of ALK + ALCL patients develop recurrent disease after first-line therapy, and they have poor outcomes and short survival. Conventional multi-agent chemotherapy regimens have been used to treat these patients as a result of low efficiency, without supportive evidence as a guide. New therapies, particularly targeted approaches, are eagerly awaited for relapsed/refractory (R/R) ALK + ALCL patients.

ALK-specific tyrosine kinase inhibitors (TKIs), such as crizotinib, which were developed for use in ALKpositive lung cancer ${ }^{1011}$, show promising therapeutic activity as salvage therapy for patients with $R / R$ $A L K+A L C L^{12-17}$. We previously reported the use of crizotinib in four R/R ALC + ALCL patients; three had a durable response and one recurred quickly ${ }^{2}$, as the resistance mechanisms are poorly understood. Fusion partners of the ALK gene vary widely and chromosomal breakpoints within the fusion gene occur at different sites, which means a difference in the fusion type of ALK gene may be the reason for resistance to current therapy. Herein, we retrospectively analyzed the characteristics and survival status of 43 ALK + ALCL patients, and the results of next-generation sequencing (NGS) for R/R patients treated with ALK TKIs.

\section{Methods}

\section{Patients}


Informed consent was received from all patients before treatment. Forty-three patients with pathologically confirmed ALCL, who were immunohistochemistry confirmed to be ALK-positive, were eligible to be included in this study. Clinical data were collected for all patients, including age, gender, Ann Arbor clinical-stage, IPI, the presence of B symptoms, bone marrow involvement, bulky disease, serum lactate dehydrogenase (LDH), and survival status. Bulky disease was defined as a tumor mass $\geq 7.5 \mathrm{~cm}$. Patients' clinical information was extracted from the electronic medical records at Guangdong Provincial People's Hospital (GPPH). This study was approved by the Institutional Review Board of GPPH.

\section{Next-generation sequencing}

Tissue samples from seven patients were sent for NGS after the crizotinib treatment. Dynamic blood samples from one patient were evaluated for circulating tumor DNA. Tissue samples obtained by biopsy were immediately placed in liquid nitrogen and stored at $-80^{\circ} \mathrm{C}$. DNA from the tissue samples was extracted using the DNeasy Blood and Tissue Kit (Qiagen, Hilden, Germany). Purified DNA was qualitatively analyzed with the Nanodrop One (Thermo Fisher Scientific, Waltham, MA, USA) and quantified with a Qubit 3.0 fluorometer (Thermo Fisher Scientific) using the dsDNA HS Assay Kit (Life Technologies, Carlsbad, CA, USA) according to the manufacturer's instructions. From 8 to $10 \mathrm{~mL}$ of peripheral blood was collected in EDTA-coated tubes during dynamic monitoring. Plasma was extracted from the peripheral blood within 2 hours. Circulating tumor DNA was extracted from the plasma using a QIAamp Circulating Nucleic Acid Kit (Qiagen). Genomic DNA of whole blood was extracted with a DNeasy Blood and Tissue Kit (Qiagen) as a germ-line control. The quantity of DNA was measured on a Qubit 3.0 fluorometer with a dsDNA HS Assay Kit (Life Technologies).

\section{Pathological diagnosis}

ALCL patients were diagnosed according to the WHO classification. This was based on both the histopathological presence of large cells with anaplastic morphology (pleomorphic nuclei, prominent nucleoli, and abundant cytoplasm) and on expression of the CD30 antigen by the tumor cells. Expression of the ALK protein was used to divide ALCL into ALK+ and ALK-subgroups.

\section{Staging and treatment}

Clinical stage was determined from a complete medical history and physical examination, computed tomography (CT) scans of the chest, abdomen, and pelvis, or positron emission tomography/computed tomography (PET/CT) scans, bone marrow aspirates, and biopsies. Patients were staged according to the Ann Arbor classification ${ }^{18}$. Three patients $<18$ years old received the BFM-NHL-90 regimen ${ }^{19}$, and the other patients received $\mathrm{CHOP}$ or $\mathrm{CHOP}$-like combination regimens containing etoposide as first-line therapies.

\section{Statistical analysis}


OS was determined from the date of diagnosis to the last contact date or death by any cause. PFS was defined as the date from diagnosis to disease progression or death by any cause, PFS ${ }^{\star}$ was defined as the date from ALK TKI treatment to disease progression or death by any cause. Survival curves were estimated using the Kaplan-Meier method, and differences between subgroups were compared using the log-rank test. The Cox proportional hazards model was used to identify independent prognostic factors. All statistical tests were two-sided, and $P$-values $<0.05$ were considered significant.

\section{Results}

\section{Patient demographics and clinicopathological profiles}

Forty-three patients with pathologically confirmed ALK+ALCL (median age, 29 years) were admitted to the Lymphoma Division at GPPH between February 2007 and February 2020. All patients were eligible and evaluable for response. Their baseline characteristics are shown in Table 1. The age cut-off value was 40 years based on a previous study. Twenty-eight patients were $<40$ years, while 15 patients were older (65.1\% vs. 34.9\%). Thirty-three patients were male with a male-female ratio of 2.9. Almost all patients were diagnosed at the advanced stage (37/43, 86\%). The frequency of the presence of B symptoms was $60.5 \%$. Sixteen patients had bulky disease. More than half of the patients $(32 / 43,74.4 \%)$ exhibited strong positive ALK expression by immunohistochemistry. Only two patients $(2 / 43,4.7 \%)$ in our study had bone marrow involvement.

\section{Survival analyses}

Forty-three patients were included in our retrospective study, with a median follow-up of 52.2 (2.4-168.6) months. Thirteen patients died of disease progression by the last follow up. The 5-year PFS and OS rates for ALK+ALCL patients were $60 \%$ and $70 \%$, respectively. The median PFS and OS of all patients were not reached. The PFS and OS survival curves are shown in Figure 1. The clinical parameters were subjected to a univariate analysis to assess their effect on PFS and OS of the entire cohort (Table 2). Age, advanced stage, elevated LDH, bulky disease, and B symptoms had no significant impact on outcomes. Bone marrow involvement and a high IPI score were associated with poor PFS and OS. To assess further the independent prognostic factors, multivariate analyses were performed using the Cox proportional hazards model, including clinical stage, serum LDH level, bulky disease, bone marrow involvement, and IPI. Only bone marrow involvement was an independent prognostic factor for PFS $(P=0.03)$ and OS $(P=$ 0.03) in ALK+ALCL patients (Table 3). Clinical stage, LDH level, B symptoms, bulky disease, bone marrow involvement, and IPI had no prognostic value for ALK+ALCL patients.

\section{Clinical characteristics of R/R ALK+ patients using TKls}

Seven patients in our cohort were treated with TKIs after failing to respond to previous therapies. Their clinicopathological features are summarized in Table 4. Of the seven patients, four were male and three were female, with a median age of 18 (range 15-51) years. All of these patients presented at an advanced stage at the initial diagnosis, and three had an elevated LDH level. The median line of therapies 
was four (range 3-7) and that of ALK inhibitor usage was three (range 2-5). An initial response was observed after 1 month in all patients (Figure 3A). The overall response rate was 7 of 7 (100\%), with six complete responses (CR) and one partial response (PR). Three patients obtained a CR on crizotinib and received an autologous stem cell transplantation, and are still complete responders. One patient obtained CR but died of serious infection 5 months after allogeneic stem cell transplantation. One patient is in CR under continuous crizotinib administration. One patient received crizotinib-obtained $\mathrm{CR}$, but 3 months later developed progression of the disease; NGS showed the TRAF1 exon 6-ALK exon 20 fusion junction. Patient 1 \# was PR on alectinib, and quickly developed resistance, with a PFS of 1.7 months. NGS indicated TRAF1 exon 7-ALK exon 20 fusion.

\section{ALK fusion type and mutant burden in patients with ALK+ALCL}

Formalin-fixed paraffin-embedded tumor tissues obtained from seven patients who received TKIs for second or third-line chemotherapy were sent for NGS. The details of ALK fusion type are shown in Table 5; ALK-TRAF1 fusion was observed in two patients (2/7), while the common NPM1-ALK fusion was observed in the remaining patients (5/7). The Integrative Genomics Viewer (Broad Institute, Cambridge, MA, USA) was used for patients 1, 2, 3, and 6 (Figure 2). A plasma sample was collected from patient 1 \# for ctDNA dynamic monitoring (Figure 4). The first-time test was run before the initial treatment for baseline value, with $14.1 \%$ mutant abundance in ALK-TRAF1 fusion. After receiving two cycles of CHOP chemotherapy, the ALK-fusion detected in the plasma decreased to only $0.1 \%$, and the efficacy evaluation was PR. ALK fusion abundance approached the baseline value at the third test, and a CT scan confirmed a disease relapse at the same time. The patient received single-agent alectinib therapy as a second-line treatment after disease relapse and ALK-TRAF1 decreased gradually. Radiological evaluation showed that the pulmonary infiltrating lymphoma lesion began to shrink after 1-week of alectinib treatment (Figure 3C). ALK p.L1196M started to appear at the seventh test immediately after recurrent disease was confirmed. The ALK p.L1196M mutant has been associated with resistance to TKIs according to recent research ${ }^{20}$.

\section{Discussion}

Although genomic annotations of ALK + ALCL have become more clarified, the mechanistic modalities of recurrent disease remain elusive. Only a few treatment options are available for R/R ALCL patients. The use of TKIs for R/R ALK + ALCL patients is becoming an option since several studies demonstrated that TKIs are a promising therapy for ALK + ALCL. The assumption remains that ALK TKI therapy is analogous across all ALCL fusions because all ALK fusion proteins retain the entire ALK kinase domain ${ }^{21}$. However, treatments are not the same in some cases. Negative correlations have been observed between kinase activity, protein stability, and the 5'ALK fusion partner, which can affect sensitivity to ALK TKIs ${ }^{22}$. Herein, we analyzed the clinical characteristics and prognostic factors in Chinese patients with ALK + ALCL from a single center and administered TKIs to seven patients with ALK-fusion R/R ALCL. The median age of the ALK + patients was 29 years in our cohort, which is similar to several previous reports ${ }^{4} 23$. The 
multivariate analysis indicated that patients with bone marrow involvement had a poor outcome compared to those without bone marrow involvement. Only two patients had bone marrow involvement in our study. It is also controversial that the IPI score was not significantly different in our study, as previous studies have demonstrated that the IPI score is a significant prognostic factor for PFS and OS ${ }^{7}$.

ALK + ALCL patients had better outcomes than the ALK-negative subtype, but they were at high risk for recurrence. High dose therapy, autologous stem cell transplantation (ASCT), or allogeneic SCT are optional for relapsed patients but a gold standard treatment is lacking ${ }^{24} 25$. The use of TKIs was effective as salvage therapy in R/R ALK + ALCL patients in our study. Six of seven achieved CR and tumor shrinkage. The TKI-based therapy showed promising antitumor activity with an overall response rate of $100 \%$. We also observed that patients who harbored the NPM1-ALK fusion would be likely to achieve CR, especially when the fusion-type was exon 4 of the NPM1 gene aligned to exon 20 of the ALK gene. Patients $1^{\#}$ and $2^{\#}$ remained in complete remission. The tumor-promoting mechanisms of different ALKfusion genes remain unclear. Zhang et al. reported that NPM1-ALK induces epigenetic silencing of the STAT5A gene, which can act as a tumor suppressor in T cell lymphoma ${ }^{26}$. Excessive amplification of NPM-ALK results in resistance to TKIs in an ALK-rearranged ALCL cell line ${ }^{27}$. They also proposed the idea, based on a preclinical model, that temporary suspension of TKI administration might benefit patients with the NPM-ALK fusion who develop resistance, which is the so-called "drug holiday" therapy ${ }^{27}$. There is no clinical evidence supporting the effectiveness of such therapy. We want to be aware of any further perspective trials focusing on TKI "drug holiday" therapy.

Patients harboring other fusion types may develop rapid drug resistance and disease recurrence. TRAF1ALK fusion was identified in patients $5^{\#}$ and $7^{\#}$. Both developed rapid disease progression after 2 months of TKI treatment. The exact function of TRAF1 is unconfirmed but it is mainly involved in intracellular signal transduction of tumor necrosis factor receptor family members ${ }^{28}$. The TRAF1-ALK fusion was first described in an ALCL patient by Feldman et al ${ }^{29}$. The patient was successfully treated with high-dose chemotherapy and ASCT. Another case report presented a 32-year-old male patient who failed autologous transplantation and crizotinib treatment ${ }^{30}$. Similar to our patients, an improvement was observed 3 months after starting crizotinib, but he failed subsequent treatment, indicating that TKI activity is short in these patients. The neoplastic phenotype may be maintained by constitutively activating the ALK and NF-KB pathways mediated by TRAF1-ALK interactions with TRAF2, according to Abate et al ${ }^{31}$. That study reported that the disease could not be completely eradicated in the patient-derived tumor graft model by administering a selective ALK inhibitor, suggesting that combining TKIs with NF-KB inhibitors may result in a favorable prognosis in those patients ${ }^{31}$. Patients with exon 5 of the NPM1 gene fusion had poor outcomes compared to those with the common NPML-ALK fusion, but we are unable to explore this due to the limited number of cases in our study. Heuckmann demonstrated that different EML4-ALK variants exhibit differential sensitivity to structurally diverse ALK kinase inhibitors, which was correlated with differences in protein stability in EML4-ALK expressing cells ${ }^{32}$. Thus, it was hypothesized that the TRAF1ALK fusion pattern has different sensitivities to TKIs. Exons 7 and 8 of the TRAFI gene fused to exon 20 
of the ALK gene identified in our patients has not been reported, while exons 5 and 6 of the TRAF1 gene fusion were reported recently ${ }^{29-31}$. In general, different kinds of fusion genes may be involved in distinct pathways or mechanisms that influence the outcomes of patients.

Liquid biopsy using the NGS assay for detecting early disease recurrence is feasible for solid tumors as well as lymphomas ${ }^{33} 34$.We presented the results of plasma ctDNA dynamic monitoring during the treatment of patient $1^{\#}$. This patient underwent two cycles of $\mathrm{CHOP}$ and the response evaluation after two cycles was PR. A CT scan performed during October 2018 revealed neoplastic lesions in the lung parenchyma combined with an infection. Bilateral pleural effusion was also observed. We documented clinical and radiological progression of disease and a failed response to CHOP. Based on the ALK-positive confirmed pathology, the patient underwent alectinib therapy and showed a sharp decrease in TRAF1-ALK fusion after a 1-month treatment. The CT evaluation showed shrinkage of the infiltrating lesion. Unfortunately, the disease progressed again. The ctDNA result showing TRAF1-ALK fusion increased as well as a new mutation in the ALK gene. We considered that both the ALK-fusion gene and the ALK p.L1196M mutant contributed to the drug resistance. When a tumor develops new resistance to a drug, it is often accompanied by new slight mutations. Thus, detecting ctDNA in the plasma might have advantages for a genetic analysis if more than one mutation can be found. The ctDNA result agreed with the radiological evaluation. Sequential detection of ctDNA in the plasma might be of benefit to change the treatment strategy, to suit the patient's new status.

In summary, we have reported the characteristics and survival status of 43 patients with ALK $+A L C L$, and the NGS results of R/R patients treated with ALK TKIs. The PFS and OS of ALK + ALCL patients were consistent with a previous study. ALK inhibitors improved survival of patients with R/R ALK-positive ALCLs. In addition, TKIs were well tolerated by all patients. Two patients achieved CR after receiving crizotinib as a second-line regimen and reached depth relief following ASCT and alloSCT. This finding suggests that the use of TKIs as a subsequent treatment before ASCT and alloSCT is recommended. TRAF1-ALK fusion may predict a poor clinical outcome to chemotherapy and ALK inhibitors, or it may reflect a trend toward the aggressive behavior of lymphomas. However, as this was a retrospective study with a small sample size, we are still uncertain about which types of ALK-fusion are most favorable for TKI treatment. Moreover, new biomarkers remain to be explored to stratify patients.

\section{Conclusion}

In conclusion, ALK inhibitors improved survival of patients with R/R ALK-positive ALCLs. Patients harboring fusion types results in different clinical outcomes and drug sensitivity. TRAF1-ALK fusion may predict a poor clinical outcome to chemotherapy and ALK inhibitors. Meanwhile TRAF1-ALK fusion may reflect a trend toward the aggressive behavior of lymphomas. Further research with larger patient cohort size and longer follow-up time is required to validate our findings. It is of urgent need to investigate the resistance mechanisms based on fusion types of ALK-positive ALCL patients. 


\section{Abbreviations}

$\mathrm{R} / \mathrm{R}$

Relapsed/Refractory

ALK

Anaplastic lymphoma kinase

ALCL

Anaplastic large cell lymphomas

TKI

Tyrosine kinase inhibitor

NPM1

nucleophosmin

TRAF1

Tumor necrosis factor receptor-associated factor 1

NGS

Next-generation sequencing

OS

Overall survival

PFS

Progression-free survival

$\mathrm{HR}$

Hazard ratio

$\mathrm{Cl}$

Confidence interval

IPI

International Prognostic Index

LDH

Lactate dehydrogenase

CT

Computed tomography

PET/CT

Positron emission tomography/Computed tomography

CR

Complete response

PR

partial response

PD

progressive disease

CHOP

Cyclophosphamide, doxorubicin, vincristine, and prednisone 
ASCT

Autologous stem cell transplantation

ctDNA

Cell-free tumor DNA

\section{Declarations}

\section{Ethics approval and consent to participate}

This study was approved by Guangdong Provincial People's Hospital and the Institutional Review Board/Ethics Committee of Guangdong Provincial People's Hospital. All patients provided informed written consent to participate prior to sample collection.

\section{Consent for publication}

Written consent was collected from each patient for future data publication.

\section{Availability of data and material}

The authors confirm that the data supporting the findings of this study are available within the article.

\section{Competing interests}

The authors declare that they have no competing interests.

\section{Funding}

This study was supported by the Science and Technology Planning Project Foundation of Guangdong Province, China (No. 2016A0505028)

\section{Authors' contributions}

DP, LH and XJ conceived and designed the study. DP, HL, XJ, FC, HG, XW and YT collected the data. DP and LH analyzed the data. SL, and ZL provided the resources for the study. WL supervised the study. DP, $\mathrm{LH}$ and XJ wrote the manuscript. All authors read and approved the final manuscript prior to submission.

\section{Acknowledgements}

We would like to thank the patients and family members who gave their consent to present data in this study, as well as the investigators and research staff. 


\section{References}

1. Laurent $\mathrm{C}$, Baron $\mathrm{M}$, Amara N, et al. Impact of expert pathologic review of lymphoma diagnosis: study of patients from the French lymphopath network. Journal of Clinical Oncology: Official Journal of the American Society of Clinical Oncology. 2017;35(18):2008-17. doi:10.1200/jco.2016.71.2083 [published Online First: 2017/05/02].

2. Huang L, Zhang F, Zeng J, et al. ALK expression plays different roles in anaplastic large-cell lymphomas and outcome of crizotinib use in relapsed/refractory ALK + patients in a Chinese population. Ann Hematol. 2018;97(1):149-59. doi:10.1007/s00277-017-3166-8 [published Online First: 2017/11/19].

3. Swerdlow SH, Campo E, Pileri SA, et al. The 2016 revision of the World Health Organization classification of lymphoid neoplasms. Blood. 2016;127(20):2375-90. doi:10.1182/blood-2016-01643569 [published Online First: 2016/03/17].

\section{4. $10.1182 /$ blood-2010-02-270785}

Schmitz N, Trümper L, Ziepert M, et al. Treatment and prognosis of mature T-cell and NK-cell lymphoma: an analysis of patients with T-cell lymphoma treated in studies of the German HighGrade Non-Hodgkin Lymphoma Study Group. Blood 2010;116(18):3418-25. doi: 10.1182/blood2010-02-270785 [published Online First: 2010/07/28].

5. Kutok JL, Aster JC. Molecular biology of anaplastic lymphoma kinase-positive anaplastic large-cell lymphoma. Journal of Clinical Oncology: Official Journal of the American Society of Clinical Oncology. 2002;20(17):3691-702. doi:10.1200/jco.2002.12.033 [published Online First: 2002/08/31].

6. Matsuyama $\mathrm{H}$, Suzuki HI, Nishimori $\mathrm{H}$, et al. miR-135b mediates NPM-ALK-driven oncogenicity and renders IL-17-producing immunophenotype to anaplastic large cell lymphoma. Blood. 2011;118(26):6881-92. doi:10.1182/blood-2011-05-354654 [published Online First: 2011/11/02].

7. Savage KJ, Harris NL, Vose JM, et al. ALK- anaplastic large-cell lymphoma is clinically and immunophenotypically different from both ALK + A LCL and peripheral T-cell lymphoma, not otherwise specified: report from the International Periphera I T-Cell Lymphoma Project. Blood. 2008;111(12):5496-504.

8. Falini B, Pileri S, Zinzani PL, et al. ALK + lymphoma: clinico-pathological findings and outcome. Blood. 1999;93(8):2697-706. [published Online First: 1999/04/09].

9. Gao J, Yin M, Zhu Y, et al. Prognostic significance and therapeutic potential of the activation of anaplastic lymphoma kinase/protein kinase B/mammalian target of rapamycin signaling pathway in anaplastic large cell lymphoma. BMC Cancer. 2013;13:471. doi:10.1186/1471-2407-13-471 [published Online First: 2013/10/12].

10. Kwak EL, Bang YJ, Camidge DR, et al. Anaplastic lymphoma kinase inhibition in non-small-cell lung cancer. N Engl J Med. 2010;363(18):1693-703. doi:10.1056/NEJMoa1006448 [published Online First: 2010/10/29]. 
11. Gainor JF, Dardaei L, Yoda S, et al. Molecular mechanisms of resistance to first- and secondGeneration ALK inhibitors in ALK-rearranged lung cancer. Cancer Discov. 2016;6(10):1118-33. doi:10.1158/2159-8290.Cd-16-0596 [published Online First: 2016/07/20].

12. Li R, Morris SW. Development of anaplastic lymphoma kinase (ALK) small-molecule inhibitors for cancer therapy. Med Res Rev. 2008;28(3):372-412. doi:10.1002/med.20109 [published Online First: 2007/08/19].

13. Gambacorti-Passerini C, Messa C, Pogliani EM. Crizotinib in anaplastic large-cell lymphoma. N Engl J Med. 2011;364(8):775-6. doi:10.1056/NEJMc1013224 [published Online First: 2011/02/25].

14. Mosse YP, Lim MS, Voss SD, et al. Safety and activity of crizotinib for paediatric patients with refractory solid tumours or anaplastic large-cell lymphoma: a Children's Oncology Group phase 1 consortium study. The Lancet Oncology. 2013;14(6):472-80. published Online First: 2013/04/20]. ( .. doi: 10.1016/s1470-2045.

15. Gambacorti Passerini C, Farina F, Stasia A, et al. Crizotinib in advanced, chemoresistant anaplastic lymphoma kinase-positive lymphoma patients. J Natl Cancer Inst. 2014;106(2):djt378. doi:10.1093/jnci/djt378 [published Online First: 2014/02/05].

16. Mossé YP, Voss SD, Lim MS, et al. Targeting ALK With Crizotinib in Pediatric Anaplastic Large Cell Lymphoma and Inflammatory Myofibroblastic Tumor: A Children's Oncology Group Study. Journal of Clinical Oncology: Official Journal of the American Society of Clinical Oncology. 2017;35(28):321521. doi:10.1200/jco.2017.73.4830 [published Online First: 2017/08/09].

17. Gambacorti-Passerini C, Orlov S, Zhang L, et al. Long-term effects of crizotinib in ALK-positive tumors (excluding NSCLC): A phase 1b open-label study. Am J Hematol. 2018;93(5):607-14. doi:10.1002/ajh.25043 [published Online First: 2018/01/21].

18. Armitage JO. Staging non-Hodgkin lymphoma. CA Cancer J Clin. 2005;55(6):368-76. doi:10.3322/canjclin.55.6.368 [published Online First: 2005/11/12].

19. 10.1182/blood.v97.12.3699

Seidemann K, Tiemann M, Schrappe M, et al. Short-pulse B-non-Hodgkin lymphoma-type chemotherapy is efficacious treatment for pediatric anaplastic large cell lymphoma: a report of the Berlin-Frankfurt-Münster Group Trial NHL-BFM 90. Blood 2001;97(12):3699 - 706. doi: 10.1182/blood.v97.12.3699 [published Online First: 2001/06/05].

20. Ceccon M, Mologni L, Giudici G, et al. Treatment efficacy and resistance mechanisms using the second-generation ALK inhibitor AP26113 in Hum an NPM-ALK-positive anaplastic large cell lymphoma. Mol Cancer Res. 2015;13(4):775-83.

21. 10.1093/annonc/mdw301

Hallberg B, Palmer RH. The role of the ALK receptor in cancer biology. Ann Oncol 2016;27 Suppl 3:iii4-iii15. doi: 10.1093/annonc/mdw301 [published Online First: 2016/08/31].

22. Childress MA, Himmelberg SM, Chen $\mathrm{H}$, et al. ALK fusion partners impact response to ALK inhibition: differential effects on sensitivity, cellular phenotypes, and biochemical properties. Mol Cancer Res. 2018;16(11):1724-36. 
23. Gascoyne RD, Aoun P, Wu D, et al. Prognostic significance of anaplastic lymphoma kinase (ALK) protein expression in adults with anaplastic large cell lymphoma. Blood. 1999;93(11):3913-21.

24. Kewalramani T, Zelenetz AD, Teruya-Feldstein J, et al. Autologous transplantation for relapsed or primary refractory peripheral T-cell lymphoma. Br J Haematol. 2006;134(2):202-7.

25. Rodriguez J, Munsell M, Yazji S, et al. Impact of high-dose chemotherapy on peripheral T-cell lymphomas. Journal of Clinical Oncology: Official Journal of the American Society of Clinical Oncology. 2001;19(17):3766-70.

26. Zhang Q, Wang HY, Liu X, et al. STAT5A is epigenetically silenced by the tyrosine kinase NPM1-ALK and acts as a tumor suppressor by reciprocally inhibiting NPM1-ALK expression. Nat Med. 2007;13(11):1341-8.

27. Ceccon M, Merlo MEB, Mologni L, et al. Excess of NPM-ALK oncogenic signaling promotes cellular apoptosis and drug dependency. Oncogene. 2016;35(29):3854-65.

28. Edilova MI, Abdul-Sater AA, Watts TH. TRAF1 Signaling in human health and disease. Front Immunol. 2018;9:2969. doi:10.3389/fimmu.2018.02969 [published Online First: 2019/01/09].

29. Feldman AL, Vasmatzis G, Asmann YW, et al. Novel TRAF1-ALK fusion identified by deep RNA sequencing of anaplastic large cell lymphoma. Genes Chromosomes Cancer. 2013;52(11):1097102.

30. Lawrence K, Berry B, Handshoe J, et al. Detection of a TRAF1-ALK fusion in an anaplastic large cell lymphoma patient with chemotherapy and AL K inhibitor-resistant disease. BMC Res Notes. 2015;8:308.

31. Abate $F$, Todaro $M$, van der Krogt JA, et al. A novel patient-derived tumorgraft model with TRAF1-ALK anaplastic large-cell lymphoma translocation. Leukemia. 2015;29(6):1390-401.

32. Heuckmann JM, Balke-Want $\mathrm{H}$, Malchers $F$, et al. Differential protein stability and ALK inhibitor sensitivity of EML4-ALK fusion variants. Clinical Cancer Research: an Official Journal of the American Association for Cancer Research. 2012;18(17):4682-90.

33. Chen $\mathrm{M}$, Zhao H. Next-generation sequencing in liquid biopsy: cancer screening and early detection. Hum Genomics. 2019;13(1):34. doi:10.1186/s40246-019-0220-8 [published Online First: 2019/08/03].

34. Rossi D, Diop F, Spaccarotella E, et al. Diffuse large B-cell lymphoma genotyping on the liquid biopsy. Blood. 2017;129(14):1947-57. doi:10.1182/blood-2016-05-719641 [published Online First: 2017/01/18].

\section{Tables}

Table 1 Baseline characteristics of the 43 ALK-positive ALCL patients 


\begin{tabular}{|c|c|}
\hline Characteristics & $\mathrm{N}(\%)$ \\
\hline Median age (range) & $29(15-66)$ \\
\hline \multicolumn{2}{|l|}{ Age } \\
\hline$\leq 40$ & $28(65.1)$ \\
\hline$>40$ & $15(34.9)$ \\
\hline \multicolumn{2}{|l|}{ Sex } \\
\hline Male & $32(74.4)$ \\
\hline Female & $11(25.6)$ \\
\hline \multicolumn{2}{|l|}{ IPI } \\
\hline $0 \sim 1$ & $13(30.2)$ \\
\hline 2 & $16(37.2)$ \\
\hline 3 & $12(27.9)$ \\
\hline $4 \sim 5$ & $2(4.7)$ \\
\hline \multicolumn{2}{|c|}{ Stage at initial diagnosis } \\
\hline I/II & $6(14.0)$ \\
\hline III/IV & $37(86.0)$ \\
\hline \multicolumn{2}{|l|}{ B symptoms } \\
\hline Yes & $26(60.5)$ \\
\hline No & $17(39.5)$ \\
\hline \multicolumn{2}{|l|}{ Bulky disease } \\
\hline Yes & $16(37.2)$ \\
\hline No & $27(62.8)$ \\
\hline \multicolumn{2}{|l|}{ Lactate dehydrogenase } \\
\hline Normal (<245 U/L) & $21(48.8)$ \\
\hline Elevated ( $\geq 245 \mathrm{U} / \mathrm{L}$ ) & $22(51.2)$ \\
\hline \multicolumn{2}{|l|}{ IHC for ALK status } \\
\hline+ & $10(23.3)$ \\
\hline++ & $1(2.3)$ \\
\hline+++ & $32(74.4)$ \\
\hline
\end{tabular}

Page 15/21 
Ki-67 expression

Positive $\geq 90 \%$

22(51.2)

Positive $<90 \%$

21(48.8)

Baseline BM involvement $2(4.7)$

IPI: international prognostic index; LDH: lactate dehydrogenase; BM: bone marrow; IHC: immunohistochemistry

Table 2 Univariate analyses of overall and progression-free survival of all patients

\begin{tabular}{|c|c|c|c|c|c|c|}
\hline \multirow{3}{*}{ Characteristic } & \multicolumn{6}{|c|}{ Univariate analysis } \\
\hline & \multicolumn{3}{|l|}{ PFS } & \multicolumn{3}{|l|}{ os } \\
\hline & $\mathrm{HR}$ & $95 \% \mathrm{Cl}$ & $P$ & HR & $95 \% \mathrm{Cl}$ & $P$ \\
\hline Age $>40$ years & 0.86 & $0.30-2.45$ & 0.78 & 1.27 & $0.42-3.88$ & 0.68 \\
\hline Stage $^{\#}$ & 3.39 & $0.45-25.63$ & 0.23 & 2.30 & $0.30-17.68$ & 3.39 \\
\hline $\mathrm{LDH} \geq 245 \mathrm{U} / \mathrm{L}$ & 0.66 & $0.25-1.76$ & 0.41 & 1.18 & $0.40-3.53$ & 0.66 \\
\hline Bulky disease & 1.52 & $0.57-4.00$ & 0.40 & 1.62 & $0.54-4.81$ & 1.52 \\
\hline B symptoms & 0.88 & $0.34-2.31$ & 0.80 & 1.08 & $0.35-3.30$ & 0.90 \\
\hline BM involvement & 5.53 & $1.22-25.05$ & 0.03 & $7 . .21$ & $1.44-36.07$ & 0.02 \\
\hline \multicolumn{7}{|l|}{ IPI score } \\
\hline 2 & 0.80 & $0.23-2.78$ & 0.73 & 1.50 & $0.28-8.02$ & 0.63 \\
\hline 3 & 1.50 & $0.43-5.20$ & 0.52 & 3.42 & $0.66-17.64$ & 0.14 \\
\hline $4-5$ & 5.30 & $0.98-28.50$ & 0.05 & 11.81 & $1.64-84.91$ & 0.01 \\
\hline Low $(0-2)$ vs. high $(3-5)$ & 2.11 & $0.80-5.56$ & 0.13 & 3.33 & $1.12-9.94$ & 0.03 \\
\hline
\end{tabular}

LDH: lactate dehydrogenase; IPI: international prognostic index; BM: bone marrow; \#Stage: I-II vs. III-IV;

Table 3 Multivariate analyses of overall and progression-free survival of all patients 


\begin{tabular}{|c|c|c|c|c|c|c|}
\hline \multirow{3}{*}{ Variables } & \multicolumn{6}{|c|}{ Multivariate analysis } \\
\hline & \multicolumn{3}{|l|}{ PFS } & \multicolumn{3}{|l|}{ OS } \\
\hline & $\mathrm{HR}$ & $95 \% \mathrm{Cl}$ & $P$ & HR & $95 \% \mathrm{Cl}$ & $P$ \\
\hline \multicolumn{7}{|l|}{ Stage } \\
\hline I-II vs. III-IV & 3.09 & $0.36-26.47$ & 0.30 & 1.61 & $0.18-14.58$ & 0.67 \\
\hline \multicolumn{7}{|l|}{ LDH level } \\
\hline Normal vs. elevated & 0.38 & $0.12-1.19$ & 0.10 & 0.43 & $0.10-1.79$ & 0.24 \\
\hline \multicolumn{7}{|l|}{ Bulky disease } \\
\hline Without vs. with & 1.97 & $0.63-6.16$ & 0.23 & 1.97 & $0.55-7.00$ & 0.30 \\
\hline \multicolumn{7}{|l|}{ BM involvement } \\
\hline Without vs. with & 5.83 & $1.16-29.43$ & 0.03 & 7.56 & $1.25-45.57$ & 0.03 \\
\hline \multicolumn{7}{|l|}{ IPI score } \\
\hline Low (0-2) vs. High (3-5) & 2.72 & $0.77-9.62$ & 0.12 & 3.98 & $0.86-18.42$ & 0.07 \\
\hline
\end{tabular}

LDH: lactate dehydrogenase; IPI: international prognostic index; BM: bone marrow

Table 4 Clinical features of seven relapsed/refractory ALK+ patients using ALK TKIs

\begin{tabular}{|c|c|c|c|c|c|c|c|c|c|c|}
\hline $\begin{array}{l}\text { Pt. } \\
\text { N }\end{array}$ & age & sex & stage & Bulky & $\begin{array}{l}\text { ALK } \\
\text { IHC }\end{array}$ & $\begin{array}{l}\text { Line of } \\
\text { TKI } \\
\text { usage }\end{array}$ & $\begin{array}{l}\text { Type } \\
\text { of } \\
\text { TKls }\end{array}$ & $\begin{array}{l}\text { Response } \\
\text { to } \\
\text { TKls }\end{array}$ & $\operatorname{PFS}^{*}(\mathrm{~m})$ & OS(m) \\
\hline 1 & 37 & $M$ & IV & no & + & second & Alectinib & PR & 1.7 & 8.5 \\
\hline 2 & 20 & $M$ & IV & yes & +++ & second & Crizotinib & CR & 2.6 & 8.0 \\
\hline 3 & 51 & $\mathrm{M}$ & IV & no & ++ & fifth & Crizotinib & CR & 3.2 & 11.0 \\
\hline 4 & 17 & $F$ & III & no & +++ & third & Crizotinib & CR & 79.3 & 96.2 \\
\hline 5 & 17 & M & IV & no & +++ & third & Crizotinib & CR & 76.1 & 94.0 \\
\hline 6 & 15 & $F$ & IV & no & +++ & third & Crizotinib & $\mathrm{CR}$ & 6.2 & 20.9 \\
\hline 7 & 26 & $M$ & IV & no & +++ & second & Crizotinib & $\mathrm{CR}$ & 38.2 & 42.2 \\
\hline
\end{tabular}

Abbreviations: Pt. N: patient number; F: female; M: male; IHC: immunohistochemistry; m: months. CR: complete response; PD: progressive disease; 
PFS*: The date from ALK TKI treatment to disease progression or death by any cause

Table 5 ALK fusion type of seven patients after TKI treatment

\begin{tabular}{|ll|}
\hline Pt. No & ALK fusion type \\
\hline 1 & TRAF1:exon7-ALK:exon20 \\
\hline 2 & NPM1:exon4-ALK:exon20 \\
\hline 3 & TRAF1:exon8-ALK:exon20 \\
\hline 5 & NPM1:exon4-ALK:exon20 \\
\hline 6 & NPM1:exon5-ALK:exon20 \\
\hline 7 & NPM1:exon4-ALK:exon20 \\
\hline
\end{tabular}

\section{Figures}

A

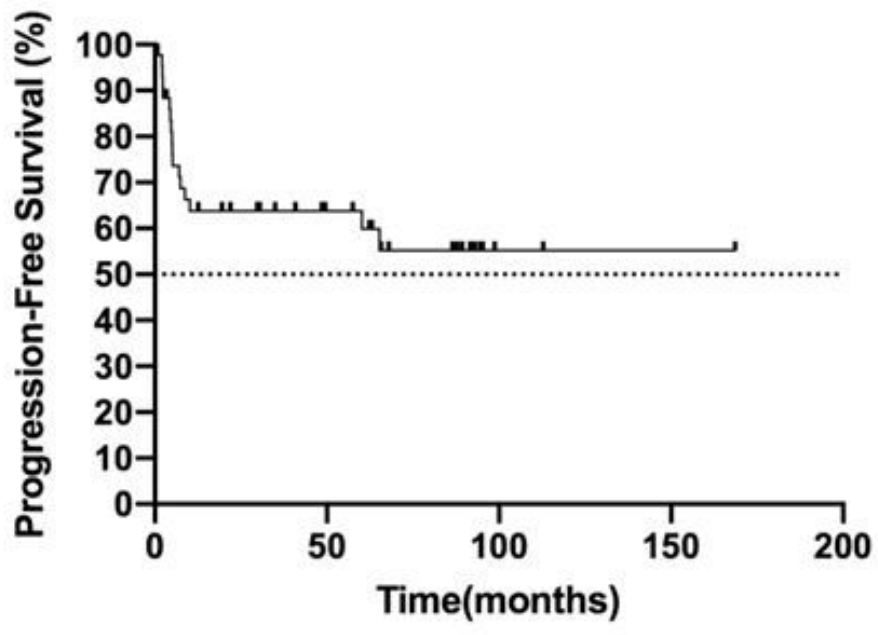

B

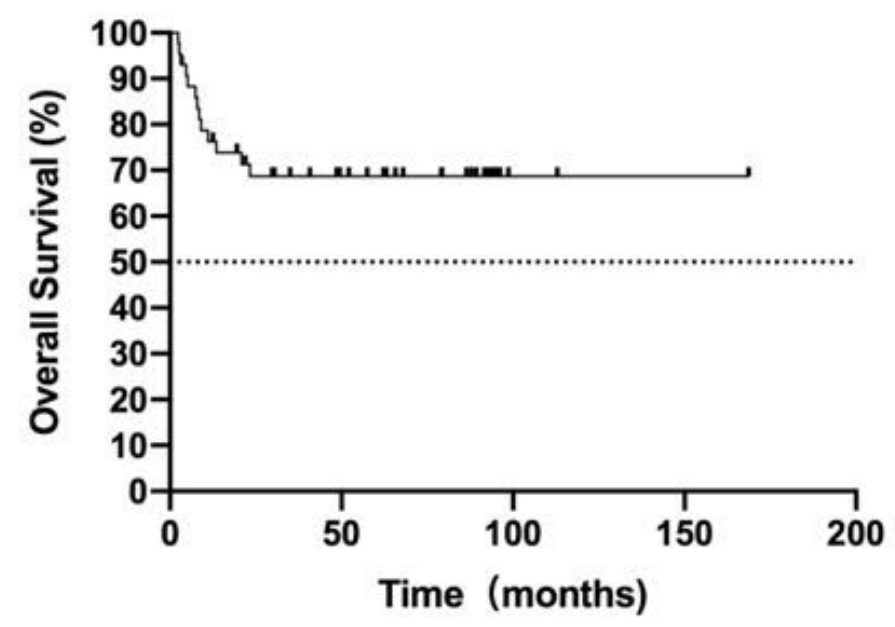

\section{Figure 1}

Progression-free survival (PFS) and overall survival (OS) of 43 ALK+ALCL patients. (A) PFS (B) OS 


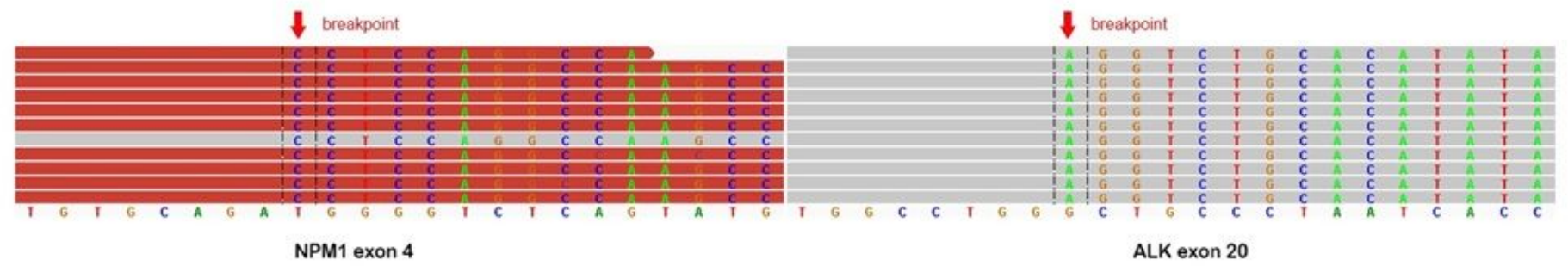

B

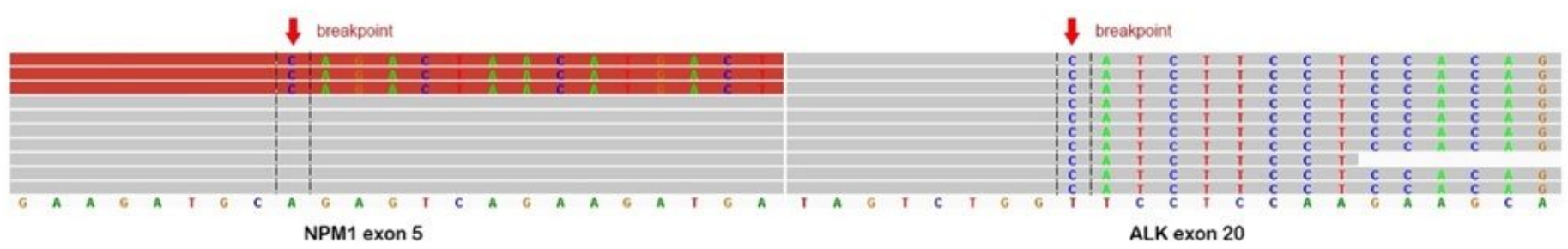

C

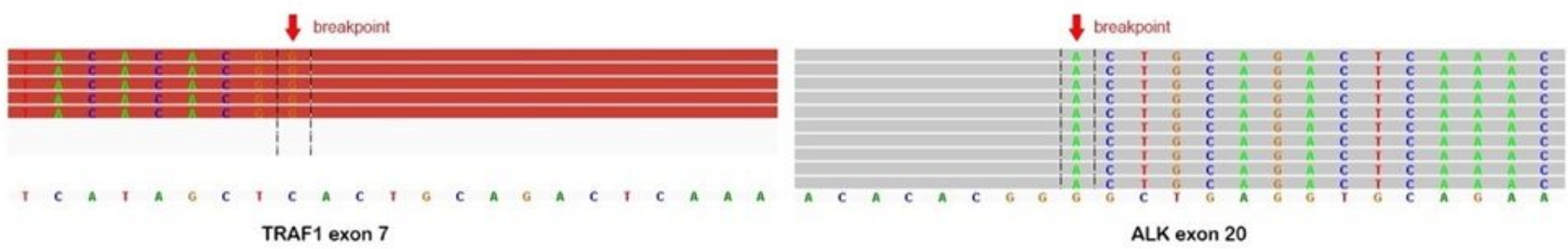

D

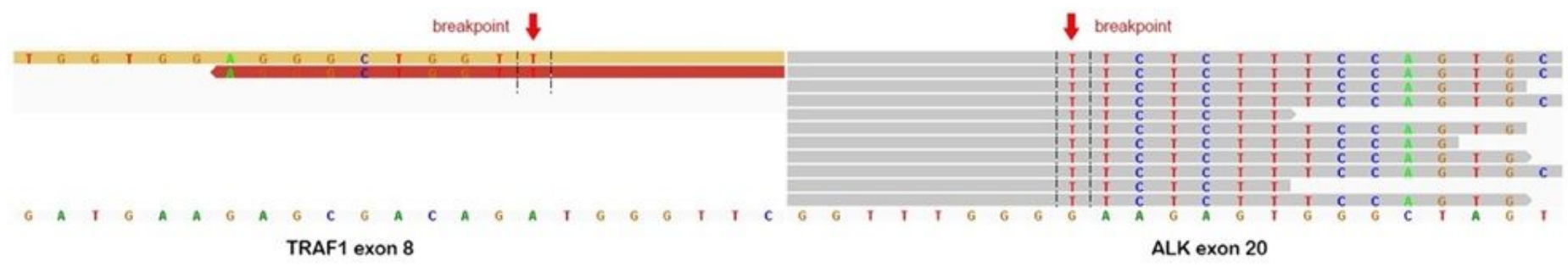

\section{Figure 2}

Sequencing reads of the ALK receptor tyrosine kinase gene (ALK) and nucleophosmin 1 gene (NPM1) in patient 2\# (A) and patient 6\# (B) were visualized with the Integrative Genomics Viewer as well as the tumor necrosis factor receptor-associated factor 1 gene (TRAF1) in patient 1\# (C) and patient 3\# (D). The sequencing reads in the ALK exon 20 region that aligned to a different gene locus are color-coded, with bases marked to identify the other loci mated to NPM1 and the TRAF1 region. 


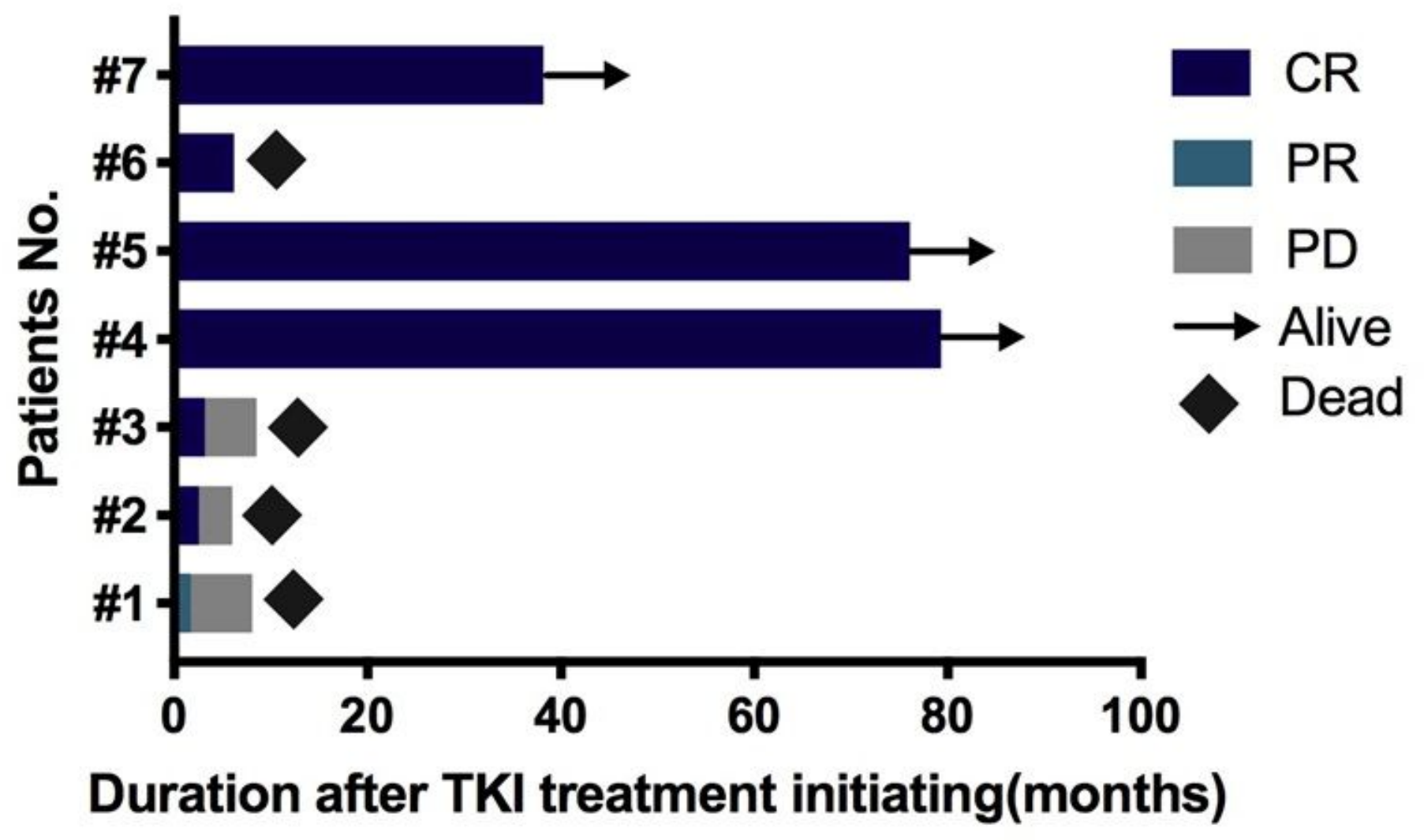

B
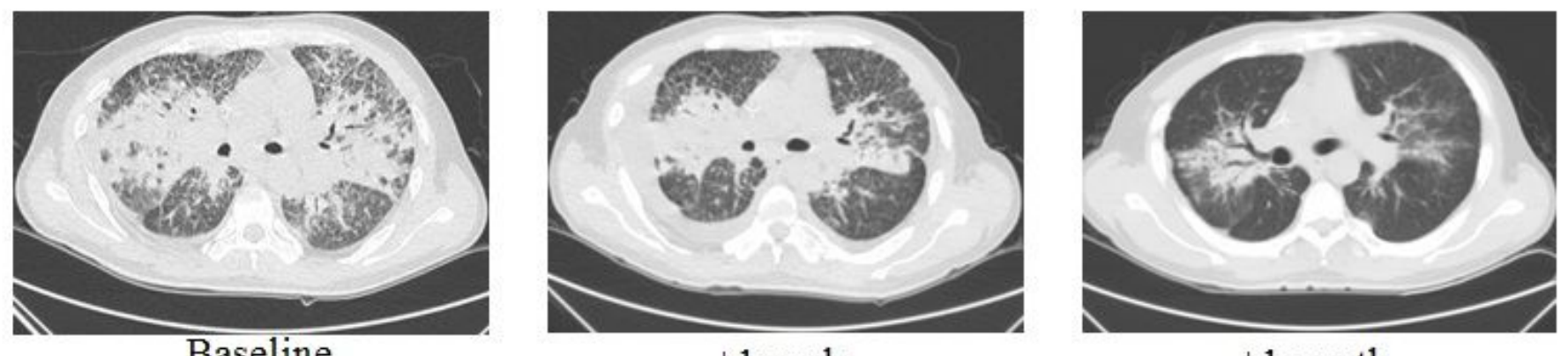

+1 week

$+1 \mathrm{month}$

Figure 3

Clinical response to TKIs in R/R ALK+ ALCL. (A) Summary of patients' progression-free survival treated with TKIs. (B) Radiological evaluation 1 week and 1 month after alectinib treatment in patient $1 \#$. 


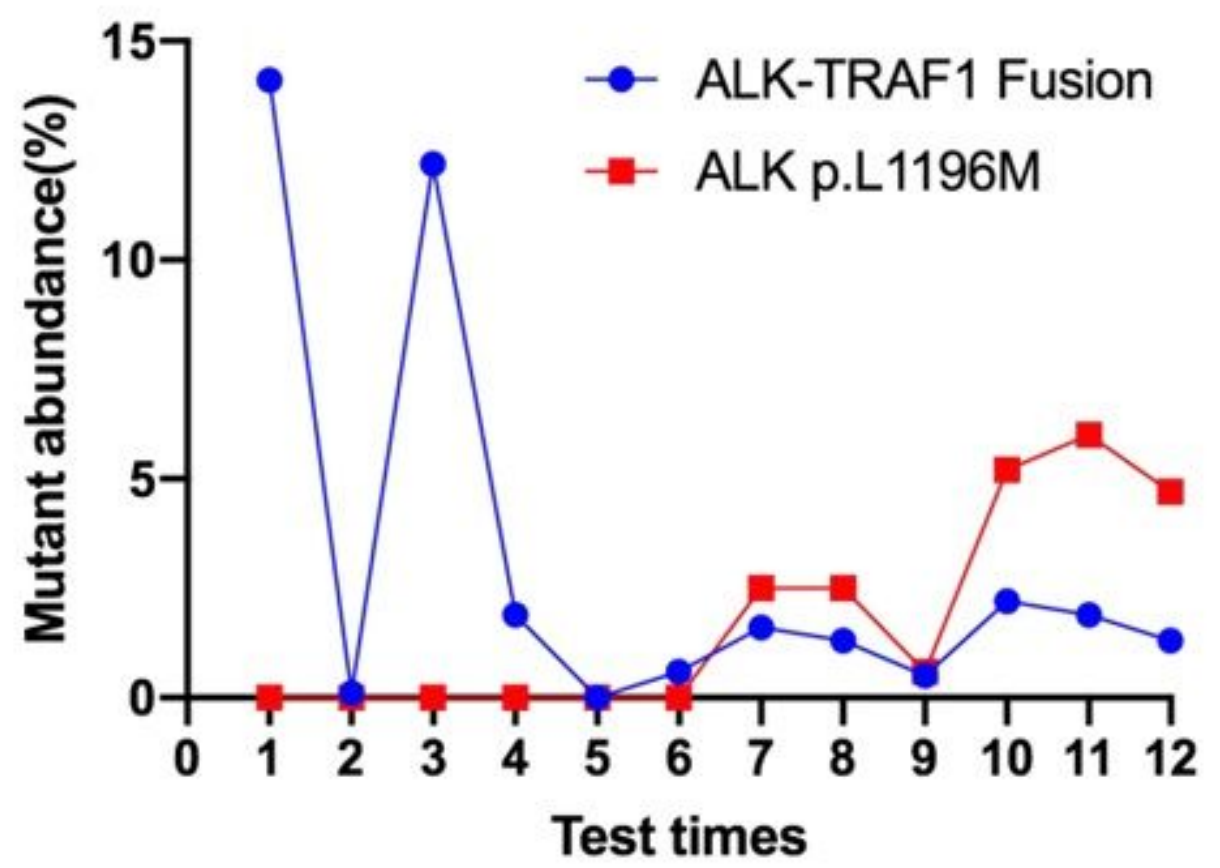

Figure 4

Dynamic monitoring of circulating tumor DNA using next-generation sequencing in plasma from patient $1 \#$. 